\title{
On the anticyclotomic Iwasawa theory of CM forms at supersingular primes
}

\author{
Kâzım Büyükboduk
}

\begin{abstract}
In this paper, we study the anticyclotomic Iwasawa theory of a CM form $f$ of even weight $w \geq 2$ at a supersingular prime, generalizing the results in weight 2, due to Agboola and Howard. In due course, we are naturally lead to a conjecture on universal norms that generalizes a theorem of Perrin-Riou and Berger and another that generalizes a conjecture of Rubin (the latter seems linked to the local divisibility of Heegner points). Assuming the truth of these conjectures, we establish a formula for the variation of the sizes of the Selmer groups attached to the central critical twist of $f$ as one climbs up the anticyclotomic tower. We also prove a statement which may be regarded as a form of the anticyclotomic main conjecture (without $p$-adic $L$-functions) for the central critical twist of $f$.
\end{abstract}

\section{Introduction}

The goal of this article is to study the anticyclotomic Iwasawa theory of an elliptic newform $f$ of even weight $w \geq 2$ which has complex multiplication (CM) by an imaginary quadratic field $K$, at a supersingular prime $p$ and thereby extend the results of Agboola and Howard [1] where the authors have studied the case $w=2$. The results of this paper also should be considered as the first steps towards an anticyclotomic main conjecture for CM forms in the supersingular setting, where the analogous results when $p$ is an ordinary prime for $f$ have been obtained by Arnold [2].

There are two main ingredients that go into the proof of the results presented in this article:

(1) The construction and analysis of \pm -Selmer groups, in the spirit of Kobayashi [18].

(2) The proof that a central critical twist of the elliptic unit Euler system along the anticyclotomic Iwasawa tower is nonvanishing; and that this Euler system controls the \pm -Selmer groups constructed in step (1).

Mathematics Subject Classification (2010): Primary 11R23, 11F11, 11G40; Secondary 14 G10. Keywords: CM modular forms, anticyclotomic Iwasawa theory. 
Although, generally speaking, the approach here relies on the arguments of [1], one runs into serious difficulty if one attempts to carry out steps (1) and (2) above when $w \neq 2$. We first explain the difficulties that one encounters and sketch how we attempt to resolve them in this paper.

First, when the weight is 2, Agboola and Howard have the formal group of the associated elliptic curve at their disposal to analyze the local cohomology groups. Their approach does not apply in the general setting, and so one is led to appeal to $p$-adic Hodge theory to carry out step (1) above; see Section 2 below. The desired properties for the \pm -subgroups of the local cohomology groups all then follow from suitable generalizations (which unfortunately we are unable to prove) of the following fundamental results. The first (Conjecture 2.5) is a statement about the universal norms along the anticyclotomic tower and an extension of a conjecture of Nekovár (on the universal norms along the cyclotomic tower) which was proved by Perrin-Riou [27] and Berger [5]; it was also proved by Rubin [29] along the anticyclotomic tower when the weight is 2. The second (Conjecture 2.13) is a generalization of Conjecture 2.2 in [30], and Rubin proved his conjecture when the weight is 2 under some assumptions on the prime $p$, by relating it to the local divisibility of Heegner points. It would be interesting to know if Nekováŕs's [22] Heegner cycles on Kuga-Sato varieties could play a role along these line to prove Conjecture 2.13 under hypotheses similar to those of Theorem 8.4 in [30].

In order to carry out step (2), as we do not have the nonvanishing results of [28] for higher weight CM forms, we adapt instead the method of Arnold [2] to prove the nontriviality of the twisted elliptic unit Euler system along the anticyclotomic tower, which in turn is based on a nonvanishing theorem of Greenberg [13]. We then relate the twisted elliptic unit Euler system to Kato's Euler system of Beilinson elements (see Section 15.16.1 of [17]) and make use of Kato's explicit reciprocity laws to calculate the image of Kato-Beilinson elements under the dual exponential map in terms of the relevant $L$-values.

Once we overcome these difficulties to settle steps (1) and (2) above, the main results of the paper follow as a standard application of the Euler system machinery. Before we state our results more precisely, we introduce some notation.

\subsection{Notation and hypotheses}

Let $f=\sum a_{n} q^{n} \in S_{w}(N, \epsilon)$ be a normalized eigenform of even weight $w \geq 2$, level $N$, and character $\epsilon$, and let $\bar{f}=\sum \bar{a}_{n} q^{n}$ be the dual form. Fix a prime $p>3$ such that $p \nmid N$ and $a_{p}=0$. Let $F=\mathbb{Q}\left(\left\{a_{n}\right\}\right)$ be the number field generated by the Fourier coefficients of $f$ (equivalently, by those of $\bar{f}$ ). Fix a completion $\Phi$ of $F$ at a prime above $p$ and let $\rho_{f}$ denote the 2-dimensional $\Phi$-representation of $G_{\mathbb{Q}}:=\operatorname{Gal}(\overline{\mathbb{Q}} / \mathbb{Q})$ attached to $f$ by Deligne $[10]$. Let $V_{f}$ denote the 2-dimensional $\Phi$-vector space that realizes $\rho_{f}$. Then for any integer $r$,

$$
V_{\bar{f}}(r) \cong \operatorname{Hom}\left(V_{f}(w-r), \Phi(1)\right)
$$

as $G_{\mathbb{Q}^{-}}$-representations, where $\Phi(1)=\Phi \otimes_{\mathbb{Z}_{p}}\left(\lim _{\boldsymbol{\mu}_{p^{n}}}\right)$ and $\boldsymbol{\mu}_{p^{n}}$ denotes the group of $p^{n}$-th roots of unity; see [17], Section 14.10 . 
Throughout, we will assume that $F=\mathbb{Q}$ (it should be possible to relax this assumption with some work). Note in this case that $f=\bar{f}$ and (1.1) implies that the $\mathbb{Q}_{p}$-representation $V_{f}(w / 2)$ is self-dual (in the sense that $V_{f}(w / 2) \cong$ $\left.\operatorname{Hom}\left(V_{f}(w / 2), \mathbb{Q}_{p}(1)\right)\right)$. Let $T_{f}$ be a fixed $\mathbb{Z}_{p}$-lattice inside $V_{f}$.

The eigenform $f$ is called a CM form if there exists an imaginary quadratic field $K$ and an algebraic Hecke character

$$
\psi: \mathbb{A}_{K} / K^{\times} \longrightarrow \mathbb{C}^{\times}
$$

such that $f$ is the cusp form associated with $\psi$ (see Section 15.10 of [17]). We assume until the end of this article that $f$ is a $C M$ form and that $K$ has class number 1 . The assumption on the class number is to ensure that the properties of elliptic units which we rely on in Section 3 hold true. Let $\tau$ denote the involution on $G_{K}=\operatorname{Gal}(\bar{K} / K)$ induced by complex conjugation. As in $\S 2.1$ of [2], we assume further that $\bar{\psi} \circ \tau=\psi$, so that the $\operatorname{sign} W(\psi) \in\{ \pm 1\}$ of the functional equation of $\psi$ (namely, the sign of the functional equation for the Hecke $L$-series attached to $f$ ) makes sense.

For a prime $p \nmid N$, the condition that $a_{p}=0$ forces the prime $p$ to remain inert in $K$, for otherwise if $p=\wp \bar{\wp}$ split in $K$, then $a_{p}=\psi(\wp)+\psi(\bar{\wp})$ would be a $p$-adic unit. Write $\mathfrak{p}$ for the unique prime of $K$ above $p$. Let $K_{\mathfrak{p}}$ be its completion at $\mathfrak{p}$ and let $\mathcal{O}_{\mathfrak{p}}$ be its ring of integers at $\mathfrak{p}$. Denote the conductor of $\psi$ by $\mathfrak{f}=\mathfrak{f}_{\psi}$ and set $K\left(\mathfrak{f} p^{\infty}\right)=\cup_{n} K\left(\mathfrak{f} p^{n}\right)$, where for an integral ideal $\mathfrak{a}$ of $K, K(\mathfrak{a})$ denotes the ray class field of $K$ of conductor $\mathfrak{a}$. By the theory of complex multiplication, the action of $G_{\mathbb{Q}}$ on $V_{f}$ factors through $\operatorname{Gal}\left(K\left(\mathfrak{f} p^{\infty}\right) / \mathbb{Q}\right)$. The action of $G_{K}$ on the one-dimensional $K_{\mathfrak{p}}$-vector space $V_{f}$ is given by the $\mathfrak{p}$-adic avatar $\psi_{\mathfrak{p}}: G_{K} \rightarrow \mathcal{O}_{\mathfrak{p}}^{\times}$ of the Hecke character $\psi$.

Set $\boldsymbol{\mu}_{p^{\infty}}=\lim \boldsymbol{\mu}_{p^{n}}$. Following [17], let $V=V_{f}(w / 2)$ denote the central critical twist of $V_{f}$ and let $T=T_{f}(w / 2)$. Set $W=V / T$. Let $V^{*}=\operatorname{Hom}\left(V, \mathbb{Q}_{p}(1)\right)$, $T^{*}=\operatorname{Hom}\left(W, \boldsymbol{\mu}_{p^{\infty}}\right)$ and $W^{*}=\operatorname{Hom}\left(T, \boldsymbol{\mu}_{p^{\infty}}\right)$. Observe that $V \cong V^{*}$ as $G_{\mathbb{Q}^{-}}$ representations (since we assumed $F=\mathbb{Q}$ ), however we choose to distinguish them to avoid confusion in our arguments below.

Let $D_{\infty}$ denote the anticyclotomic $\mathbb{Z}_{p}$-extension of $K$ and let $D_{n}$ denote its $n$th layer. Let $\Gamma=\operatorname{Gal}\left(D_{\infty} / K\right)$ and $\Lambda=\mathcal{O}_{\mathfrak{p}}[[\Gamma]]$. For a torsion $\Lambda$-module $M$, let $\operatorname{char}(M)$ denote its characteristic ideal.

\subsection{Statements of the results}

In the statement of Theorem $\mathrm{A}$ and nowhere else in this paper, $\varphi$ denotes the Euler function. Let $f$ be a CM-form as above and let $\operatorname{Sel}\left(f / D_{n}\right)$ denote the $\mathfrak{p}$-adic Selmer group attached to central critical twist of $V_{f}$ (see Section 2.5 below for the precise definition) over $D_{n}$.

Theorem A. Assume the validity of Conjectures 2.5 and 2.13 (to which we have also alluded above). There is an integer e independent of $n$ such that, for all $n \gg 0$,

$$
\operatorname{corank}_{\mathcal{O}_{\mathfrak{p}}}\left(\operatorname{Sel}\left(f / D_{n}\right)\right)=e+\sum_{\substack{1 \leq k \leq n \\(-1)^{k}=\varepsilon}} \varphi\left(p^{k}\right) .
$$


See Theorem 4.1 below for a proof of this statement, where we in fact prove considerably more. This is a theorem of Agboola and Howard (and originally a conjecture of Greenberg, [12], page 247) when $f$ is of weight 2; it is proved unconditionally in that case. We are unfortunately forced to impose the additional hypotheses in order to prove Theorem A because:

- We lack a higher weight analogue of a nonvanishing result due to Rohrlich and in order to circumvent this problem, we appeal to the main theorem of [13]. This is precisely the point where we need Conjecture 2.13.

- The vanishing of the inverse limit of the Bloch-Kato subgroups along the anticyclotomic tower has not been established. In fact, the author's correspondence with some experts in $p$-adic Hodge theory gave him the impression that this vanishing problem has not been studied. The analogous question along the cyclotomic $\mathbb{Z}_{p}$-tower has been answered by Perrin-Riou [27] and for any $\mathbb{Z}_{p}$-tower, although only in the case when $f$ is of weight 2 (by Rubin [29]).

Let $\varepsilon$ denote the sign of $W(\psi)$. Let $\operatorname{Sel}_{-\varepsilon}\left(f / D_{\infty}\right)$ denote the Iwasawa theoretic \pm -Selmer group attached to the central critical twist of $V_{f}$ along the anticyclotomic tower; see Section 2.5 for its definition. Let $X_{-\varepsilon}\left(f / D_{\infty}\right)$ denote its Pontrygain dual, on which $\Lambda$ acts according to our convention in Definition 3.3 below. Let $\mathcal{H}_{\varepsilon}^{1} \subset \lim H^{1}\left(D_{n, \mathfrak{p}}, T^{*}\right)$ denote a certain submodule which defined in Section 2.4. Let $\mathcal{C}\left(\overleftarrow{D}_{\infty}\right) \subset \mathcal{H}_{\varepsilon}^{1}$ denote the submodule obtained from twisted elliptic units, as defined in Section 3.

Theorem B. Under the assumptions of Theorem A,

$$
\operatorname{char}\left(X_{-\varepsilon}\left(f / D_{\infty}\right)\right)=\operatorname{char}\left(\mathcal{H}_{\varepsilon}^{1} / \mathcal{C}\left(D_{\infty}\right)\right)
$$

This is Theorem 4.2 in the main text. One expects that the right side of the equality in Theorem B relates to an appropriately defined anticyclotomic $p$-adic $L$-function, via Kato's explicit reciprocity law; see Theorem 4.3 in [1] for the relevant discussion in the case $w=2$.

Remark 1.1. In case the form $f$ is nonCM, $p$-ordinary and has weight 2 , see [3], [4], [14], [15], and [21] for results on the anticyclotomic main conjecture for $f$ (where the work in [15], [21] concerns Hilbert modular forms of parallel weight). In [11], Darmon and Iovita studied the anticyclotomic Iwasawa theory of a nonCM form of weight 2 for a supersingular prime $p$. The recent work of Chida and Hsieh [9] treats the anticyclotomic main conjecture for a nonCM $p$-ordinary form $f$ of general weight $k$.

Acknowledgements. I would like to express my gratitude to Denis Benois, Laurent Berger, Brian Conrad, Antonio Lei, and Jan Nekovár̆ for helpful conversations. I also thank the anonymous referee for their suggestions to improve the exposition and pointing out several inaccuracies in the text. Parts of this work has been carried out during my repeated visits to the Pontificia Universidad Católica de Chile in Santiago, whose faculty I thank heartily for their hospitality. 


\section{Selmer groups}

\subsection{Some local analysis}

Let $\mathcal{V}$ be a crystalline representation of $G_{K_{\mathfrak{p}}}$. For a finite extension $F$ of $K_{\mathfrak{p}}$, let $\mathrm{k}_{F}$ denote its residue field and let $F_{0}$ denote the fraction field of the Witt vectors $W\left(\mathrm{k}_{F}\right)$ of $\mathrm{k}_{F}$. Let $D_{F}(\mathcal{V}):=\left(B_{\text {cris }} \otimes \mathcal{V}\right)^{G_{F}}$ denote the Dieudonné module of $\mathcal{V}$. We write $D(\mathcal{V})$ in place of the $K_{\mathfrak{p}}$-vector space $D_{K_{\mathfrak{p}}}(\mathcal{V})$. Let $\left\{D^{i}(\mathcal{V})\right\}$ denote the de Rham filtration on $D(\mathcal{V})$ and let

$$
\exp _{n, \mathcal{V}}: D_{n, \mathfrak{p}} \otimes D(\mathcal{V}) / D^{0}(\mathcal{V}) \longrightarrow H^{1}\left(D_{n, \mathfrak{p}}, \mathcal{V}\right)
$$

denote the $K_{\mathfrak{p}}\left[\Gamma_{n}\right]$-equivariant Bloch-Kato exponential map, defined as in [6]. If one assumes

$$
\begin{aligned}
& \text { the eigenvalues of the crystalline Frobenius } \varphi \text { on } D(\mathcal{V}) \text { are not } \\
& \text { integral powers of } p \text {, }
\end{aligned}
$$

then as explained in Theorem 4.1 of [6], the exponential map

$$
\exp _{n, \mathcal{V}(j)}: D_{n, \mathfrak{p}} \otimes D(\mathcal{V}(j)) / D^{0}(\mathcal{V}(j)) \hookrightarrow H^{1}\left(D_{n, \mathfrak{p}}, \mathcal{V}(j)\right)
$$

is in fact an injection for every integer $j$, and its image is denoted $H_{f}^{1}\left(D_{n, \mathfrak{p}}, \mathcal{V}(j)\right)$.

The representation $V_{f}$ attached to $f$ is crystalline and its de Rham filtration is given by

$$
D^{i}\left(V_{f}\right)=\left\{\begin{array}{ccrl}
D\left(V_{f}\right), & & i \leq 0, \\
K_{\mathfrak{p}} \omega, & 1 \leq i & \leq w-1, \\
0, & & i \geq k,
\end{array}\right.
$$

where $0 \neq \omega \in D\left(V_{f}\right)$. The action $\varphi$ of the crystalline Frobenius satisfies $\varphi^{2}+$ $\varepsilon(p) p^{w-1}=0$ (as $\left.a_{p}=0\right)$. Since we assumed that the weight $w$ is even, we conclude that $V_{f}$ satisfies the hypothesis (2.1). In particular, the Bloch-Kato exponential map induces a $K_{\mathfrak{p}}\left[\Gamma_{n}\right]$-isomorphism

$$
\exp _{n, V}: D_{n, \mathfrak{p}} \otimes D(V) / D^{0}(V) \stackrel{\sim}{\longrightarrow} H_{f}^{1}\left(D_{n, \mathfrak{p}}, V\right)
$$

for the central critical twist $V$. Fixing a choice of $\omega$ as above, we obtain an isomorphism

$$
\exp _{n, V}^{(\omega)}: D_{n, \mathfrak{p}} \stackrel{\sim}{\longrightarrow} H_{f}^{1}\left(D_{n, \mathfrak{p}}, V\right)
$$

of $K_{\mathfrak{p}}\left[\Gamma_{n}\right]$-modules, where the corestriction map

$$
\operatorname{cor}_{D_{m, \mathfrak{p}} / D_{n, \mathfrak{p}}}: H_{f}^{1}\left(D_{m, \mathfrak{p}}, V\right) \longrightarrow H_{f}^{1}\left(D_{n, \mathfrak{p}}, V\right)
$$

for $m \geq n$ corresponds to the trace map

$$
\operatorname{Tr}_{m / n}: D_{m, \mathfrak{p}} \longrightarrow D_{n, \mathfrak{p}}
$$

on the left. 
We define $H_{f}^{1}\left(D_{n, \mathfrak{p}}, W\right)$ (respectively $H_{f}^{1}\left(D_{n, \mathfrak{p}}, T\right)$ ) as the direct (respectively inverse) image of $H_{f}^{1}\left(D_{n, \mathfrak{p}}, V\right)$ under the natural map induced from the exact sequence

$$
0 \longrightarrow T \longrightarrow V \longrightarrow W \longrightarrow 0 \text {. }
$$

Define $H_{f}^{1}\left(D_{n, \mathfrak{p}}, T^{*}\right)$ (respectively $\left.H_{f}^{1}\left(K, W^{*}\right)\right)$ to be the orthogonal complement of $H_{f}^{1}\left(D_{n, \mathfrak{p}}, W\right)$ (respectively of $H_{f}^{1}\left(D_{n, \mathfrak{p}}, T\right)$ ) under the local Tate pairing.

Lemma 2.1. $H^{0}\left(D_{\infty, \mathfrak{p}}, W\right)=0=H^{0}\left(D_{\infty, \mathfrak{p}}, W^{*}\right)$.

Proof. The proof that $H^{0}\left(K_{\mathfrak{p}}, W\right)=0=H^{0}\left(K_{\mathfrak{p}}, W^{*}\right)$ follows from the proof of Lemma 4.4 in [20]. As $\Gamma_{n}$ is a nontrivial $p$-group for $n \geq 1$, we observe that

$$
\# H^{0}\left(D_{n, \mathfrak{p}}, X\right) \equiv \# H^{0}\left(K_{\mathfrak{p}}, X\right) \bmod p
$$

for $X=W, W^{*}$. The proof of the lemma follows.

\subsection{A conjecture on universal norms}

Let $K_{\text {cyc }}$ denote the cyclotomic $\mathbb{Z}_{p}$-extension of $K$ and let $K_{n}$ be its $n$th layer. It follows from Theorem 0.6 in [27] that

$$
\stackrel{\lim }{\longrightarrow} H_{f}^{1}\left(K_{n, \mathfrak{p}}, W\right)=\underline{\lim _{\longrightarrow}} H^{1}\left(K_{n, \mathfrak{p}}, W\right),
$$

or equivalently, that

$$
\lim _{\longleftarrow} H_{f}^{1}\left(K_{n, \mathfrak{p}}, T^{*}\right)=0,
$$

generalizing Theorem 2.1 in [29] (only along the cyclotomic $\mathbb{Z}_{p}$-tower); see also Lemma 7.1 in [20]. The following could be thought of as a relative version of Lei's result and can be proved without much difficulty:

Lemma 2.2. For any integer $m$,

$$
{\underset{\lim }{n}}_{n} H_{f}^{1}\left(D_{m, \mathfrak{p}} K_{n, \mathfrak{p}}, T^{*}\right)=0 \text {. }
$$

Proof. By the definition of Bloch-Kato subgroups, we have the commutative diagram with exact rows,

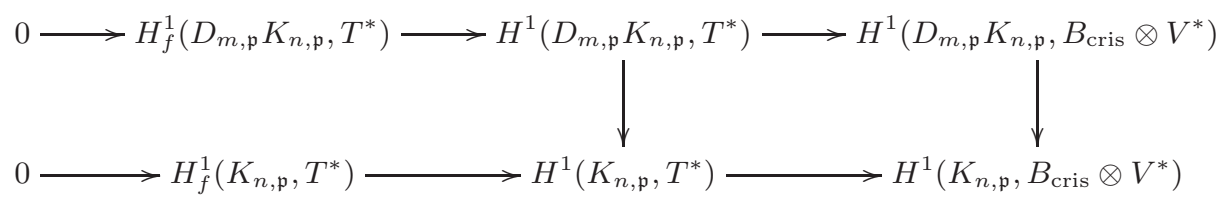

where the vertical arrows are corestriction maps. We therefore have an induced map

$$
H_{f}^{1}\left(D_{m, \mathfrak{p}} K_{n, \mathfrak{p}}, T^{*}\right) \longrightarrow H_{f}^{1}\left(K_{n, \mathfrak{p}}, T^{*}\right)
$$


Consider the map

$$
\varliminf_{s} H^{1}\left(D_{s, \mathfrak{p}} K_{n, \mathfrak{p}}, T^{*}\right) \longrightarrow H^{1}\left(D_{m, \mathfrak{p}} K_{n, \mathfrak{p}}, T^{*}\right),
$$

whose cokernel is $H^{2}\left(K_{n, \mathfrak{p}}, T^{*} \otimes \Lambda\right)\left[\gamma^{p^{m}}-1\right]$. It follows from the proof of Lemma 4.4 in [20] that $H^{0}\left(K_{n, \mathfrak{p}}, W\right)=0$. By local duality, this means $H^{2}\left(K_{n, \mathfrak{p}}, T^{*}\right)=0$, which by Nakayama's Lemma (together with the fact that the cohomological dimension of $G_{K_{n, \mathfrak{p}}}$ is 2) shows that $H^{2}\left(K_{n, \mathfrak{p}}, T^{*} \otimes \Lambda\right)=0$. We have therefore proved that the map (2.3) is surjective. This shows that the map (therefore also the map (2.2))

$$
H^{1}\left(D_{m, \mathfrak{p}} K_{n, \mathfrak{p}}, T^{*}\right) \longrightarrow H^{1}\left(K_{n, \mathfrak{p}}, T^{*}\right)
$$

is induced from reduction modulo $\gamma-1$. Thus, the map (2.2) factors through

$$
\phi_{n}: H_{f}^{1}\left(D_{m, \mathfrak{p}} K_{n, \mathfrak{p}}, T^{*}\right) /(\gamma-1) \longrightarrow H_{f}^{1}\left(K_{n, \mathfrak{p}}, T^{*}\right) .
$$

The proof follows upon passing to the inverse limit with respect to $n$ and making use of Lei's result that $\lim _{\longleftarrow} H_{f}^{1}\left(K_{n, \mathfrak{p}}, T^{*}\right)=0$ together with Nakayama's lemma.

Remark 2.3. To give this rather simple proof of Lemma 2.2, we rely on the fact that the $G_{K_{\mathrm{p}}}$-representation $V^{*}$ is irreducible and has a nonpositive Hodge-Tate weight. More generally, one can compute ${\underset{\varliminf}{\longleftarrow}}_{n} H_{f}^{1}\left(D_{m, \mathfrak{p}} K_{n, \mathfrak{p}}, T^{*}\right)$ using [5] even in the absence of this strong assumption.

Note that Lemma 2.2 is essential for the definition of a Kummer pairing as in $\S 7.3$ of [20], based on which one can prove the following explicit reciprocity law. Let $\Phi$ be any finite extension of $\mathbb{Q}_{p}$. By Kummer theory, $H^{1}\left(\Phi, \mathcal{O}_{\mathfrak{p}}(1)\right)$ can be identified with $\widehat{\Phi^{\times}} \otimes_{\mathbb{Z}_{p}} \mathcal{O}_{\mathfrak{p}}$ where $\widehat{M}$ stands for the $p$-adic completion of an abelian group $M$. Let $U_{\Phi} \subset \widehat{\Phi^{\times}} \otimes_{\mathbb{Z}_{p}} \mathcal{O}_{\mathfrak{p}}$ denote the submodule generated by the completion of units. There is a twisting isomorphism

$$
{\underset{\lim }{\Phi}}_{\Phi} H^{1}\left(\Phi, \mathcal{O}_{\mathfrak{p}}(1)\right) \stackrel{\sim}{\sim} \stackrel{\lim }{\Phi} H^{1}\left(\Phi, T^{*}\right)
$$

where the inverse limit is taken over finite subextensions $\Phi$ of the unique $\mathbb{Z}_{p^{-}}^{2}$ extension of $K_{\mathfrak{p}}$; see Section 6 of [32]. Denote the image of $\lim U_{\Phi}$ under this isomorphism by $\mathcal{U}$. Let $F$ be a finite subextension of $D_{\infty, \mathfrak{p}}$ and let $F_{\text {cyc }}$ be the cyclotomic $\mathbb{Z}_{p}$-extension of $F$. Let

$$
\mathcal{U}_{F_{\mathrm{cyc}}} \subset \underbrace{}_{\substack{L \stackrel{\lim }{L F_{\mathrm{cyc}}} \\ \text { finite over } F}} H^{1}\left(L, T^{*}\right)
$$

be the image of $\mathcal{U}$ under the natural projection. Set $H_{f}^{1}\left(F_{\text {cyc }}, W\right):=\lim _{\longrightarrow} H_{f}^{1}(L, W)$.

Lemma 2.4. (i) There is a Kummer pairing $\langle\rangle:, H_{f}^{1}\left(F_{\text {cyc }}, W\right) \times \mathcal{U}_{F_{\text {cyc }}} \rightarrow K_{\mathfrak{p}} / \mathcal{O}_{\mathfrak{p}}$. 
(ii) For any character $\chi$ of $\Delta=\operatorname{Gal}\left(F / K_{\mathfrak{p}}\right)$ and $x \in H_{f}^{1}(F, T)$, any nonnegative integer $m$ and $y=\left\{y_{L}\right\} \in \mathcal{U}_{F_{\text {cyc }}}$, we have

$$
\sum_{\delta \in \Delta} \chi(\delta)\left\langle x \otimes p^{-m}, y_{F}\right\rangle=p^{-m}\left[\sum_{\delta \in \Delta} \chi(\delta) \exp _{F, V}^{-1}\left(x^{\delta}\right), \sum_{\sigma \in \Delta} \chi^{-1}(\sigma) \exp _{F, V^{*}}^{*}\left(y_{F}^{\delta}\right)\right],
$$

where [, ] is the natural pairing on $(F \otimes D(V)) \times\left(F \otimes D\left(V^{*}\right)\right)$ and $\exp _{F, V}^{-1}$ is the inverse of the Bloch-Kato exponential

$$
\exp _{F, V}: F \otimes D(V) / D^{0}(V) \stackrel{\sim}{\longrightarrow} H_{f}^{1}(F, V) .
$$

Proof. See the proof of Proposition 7.8 in [20].

Conjecture 2.5. $\lim _{\longrightarrow} H_{f}^{1}\left(D_{n, \mathfrak{p}}, W\right)=\lim _{\longrightarrow} H^{1}\left(D_{n, \mathfrak{p}}, W\right)$. Equivalently,

$$
\lim _{\leftarrow} H_{f}^{1}\left(D_{n, \mathfrak{p}}, T^{*}\right)=0 \text {. }
$$

When the weight of $f$ is 2 , this is a theorem of Rubin [29]. The proof of this conjecture would follow from the following extension of a conjecture of Nekovár (proved by Perrin-Riou [27] in the crystalline case, and by Berger [5] in general) to the anticyclotomic $\mathbb{Z}_{p}$-extension (which was originally formulated for the cyclotomic $\mathbb{Z}_{p}$-tower):

Conjecture 2.6. Let $\mathcal{V}$ be a crystalline representation of $G_{K_{\mathfrak{p}}}$ and let $\mathcal{T}$ be a $G_{K_{\mathfrak{p}}}$-stable lattice. Assume that (2.1) holds, as well as that

$$
H^{0}\left(K_{\mathfrak{p}}, \mathcal{V} / \mathcal{T}\right)=0
$$

Then there is an isomorphism

$$
\lim _{\longleftarrow} H^{1}\left(D_{n, \mathfrak{p}}, \operatorname{Fil}^{1} \mathcal{T}\right) \stackrel{\sim}{\longrightarrow} \lim _{\longleftarrow} H_{f}^{1}\left(D_{n, \mathfrak{p}}, \mathcal{T}\right),
$$

where $\mathrm{Fil}^{1} \mathcal{V}$ denotes the largest subrepresentation of $\mathcal{V}$ with strictly positive HodgeTate weights and $\mathrm{Fil}^{1} \mathcal{T}=\mathcal{T} \cap \mathrm{Fil}^{1} \mathcal{V}$. In particular, if $\mathcal{V}$ is irreducible with at least one nonpositive Hodge-Tate weight then $\lim _{\longleftarrow} H_{f}^{1}\left(D_{n, \mathfrak{p}}, \mathcal{T}\right)=0$.

As a consequence of the proof of Nekovář's conjecture alluded to above, PerrinRiou (see Theorem 0.7 in [27]) deduces the following twisting result (with the notation of Conjecture 2.6):

$$
\lim _{\longleftarrow} H_{f}^{1}\left(K\left(\boldsymbol{\mu}_{p^{n}}\right)_{\mathfrak{p}}, \mathcal{T}\right) \otimes \mathbb{Z}_{p}(1) \hookrightarrow \lim _{\longleftarrow} H_{f}^{1}\left(K\left(\boldsymbol{\mu}_{p^{n}}\right)_{\mathfrak{p}}, \mathcal{T}(1)\right)
$$

As a generalization of this result to anticyclotomic setting, we propose the following (which in turn could be used to reduce Conjecture 2.5 to weight 2, which is known thanks to Rubin's work [29]):

Conjecture 2.7. For any character $\phi$ of $\Gamma$,

$$
\underset{\lim }{\longleftarrow} H_{f}^{1}\left(D_{n, \mathfrak{p}}, T^{*}\right) \otimes \phi \stackrel{\sim}{\longrightarrow} \lim _{\longleftarrow} H_{f}^{1}\left(D_{n, \mathfrak{p}}, T^{*} \otimes \phi\right) .
$$

Note that for any $\phi$ as above, both $G_{K_{\mathfrak{p}}}$-representations $V^{*}$ and $V^{*} \otimes \phi$ are irreducible and both have a nonpositive Hodge-Tate weight. 


\subsection{Plus-minus subgroups}

In this section, we give the definitions of the plus-minus subgroups, slightly modifying Lei's definition. For $n \geq 0$, let $\Xi_{n}^{-}$(respectively $\Xi_{n}^{+}$) denote the set of characters of $\Gamma_{n}$ of exact order $p^{k}$ with $k$ odd (respectively $k$ even), together with the trivial character (respectively without the trivial character). Set

$$
H_{ \pm}^{1}\left(D_{n, \mathfrak{p}}, V\right)=\left\{x \in H_{f}^{1}\left(D_{n, \mathfrak{p}}, V\right): \sum_{\sigma \in \Gamma_{n}} \chi(\sigma) x^{\sigma}=0 \text {, for any } \chi \in \Xi^{\mp}\right\}
$$

Set

$$
D_{n, \mathfrak{p}}^{ \pm}=\left\{x \in D_{n, \mathfrak{p}}: \sum_{\sigma \in \Gamma_{n}} \chi(\sigma) x^{\sigma}=0, \text { for any } \chi \in \Xi^{\mp}\right\},
$$

so that $H_{ \pm}^{1}\left(D_{n, \mathfrak{p}}, V\right)$ is the isomorphic image of $D_{n, \mathfrak{p}}^{ \pm}$under $\exp _{n, V}^{(\omega)}$.

Remark 2.8. Lei defines his plus-minus subgroups by setting

$$
\begin{aligned}
D_{n, \mathfrak{p}}^{+, \text {Lei }} & =\left\{x \in D_{n, \mathfrak{p}}: \operatorname{Tr}_{n / m+1}(x) \in D_{m, \mathfrak{p}}, \text { for } 0<m \leq n \text { even }\right\} \\
& =\left\{x \in D_{n, \mathfrak{p}}: \sum_{\sigma \in \Gamma_{n}} \chi(\sigma) x^{\sigma}=0, \text { for nontrivial } \chi \in \Xi^{-}\right\}, \\
D_{n, \mathfrak{p}}^{-, \text {Lei }} & =\left\{x \in D_{n, \mathfrak{p}}: \operatorname{Tr}_{n / m+1}(x) \in D_{m, \mathfrak{p}}, \text { for } 0<m \leq n \text { odd }\right\} \\
& =\left\{x \in D_{n, \mathfrak{p}}: \sum_{\sigma \in \Gamma_{n}} \chi(\sigma) x^{\sigma}=0, \text { for nontrivial } \chi \in \Xi^{+}\right\} .
\end{aligned}
$$

In particular, note that $D_{n, \mathfrak{p}}^{-, \text {Lei }}=D_{n, \mathfrak{p}}^{-}$and that $D_{n, \mathfrak{p}}^{+} \subsetneq D_{n, \mathfrak{p}}^{+, \text {Lei }}$.

Lemma 2.9. $D_{n, \mathfrak{p}}^{+} \cap D_{n, \mathfrak{p}}^{-}=0$ and $D_{n, \mathfrak{p}}^{+}+D_{n, \mathfrak{p}}^{-}=D_{n, \mathfrak{p}}$.

Proof. Remark 2.8 and the proof of Lemma 4.9 in [20]) shows that $D_{n, \mathfrak{p}}^{+} \cap D_{n, \mathfrak{p}}^{-}$ $\subset K_{\mathfrak{p}}$. Since $\Xi_{n}^{-}$contains the trivial character, we see that $K_{\mathfrak{p}} \cap D_{n, \mathfrak{p}}^{+}=0$. This proves the first statement. Then,

Let $\omega_{n}^{ \pm}$be the two distinguished polynomials in $\Lambda$ defined as in Section 5 of [1].

$$
\begin{aligned}
\operatorname{dim}_{K_{\mathfrak{p}}}\left(D_{n, \mathfrak{p}}^{+}+D_{n, \mathfrak{p}}^{-}\right) & =\operatorname{dim}_{K_{\mathfrak{p}}}\left(D_{n, \mathfrak{p}}^{+}\right)+\operatorname{dim}_{K_{\mathfrak{p}}}\left(D_{n, \mathfrak{p}}^{-}\right) \\
& =\operatorname{deg}\left(\omega_{n}^{+}\right)+\operatorname{deg}\left(\omega_{n}^{-}\right)=\operatorname{dim}_{K_{\mathfrak{p}}}\left(D_{n, \mathfrak{p}}\right),
\end{aligned}
$$

where the second equality is explained in Remark 2.10 below.

Remark 2.10. It is straightforward to see that $\omega_{n}^{\mp}(\gamma) D_{n, \mathfrak{p}} \subset D_{n, \mathfrak{p}}^{ \pm}$. In this remark we explain why this inequality is in fact an equality. Set $\omega_{n}(\gamma):=\omega_{n}^{+}(\gamma) \omega_{n}^{-}(\gamma)=$ $\gamma^{p^{n}}-1$. Using the the fact that $\omega_{n}^{ \pm}$are both distinguished in the unique factorization domain $\Lambda$, we conclude that

$$
\omega_{n}^{\mp}(\gamma) D_{n, \mathfrak{p}}=\omega_{n}^{\mp}(\gamma) K_{\mathfrak{p}}\left[\Gamma_{n}\right]=K_{\mathfrak{p}} \otimes \omega_{n}^{\mp} \Lambda / \omega_{n}=K_{\mathfrak{p}} \otimes \Lambda / \omega_{n}^{ \pm} .
$$


Thus $\operatorname{dim} D_{n, \mathfrak{p}}^{ \pm} \geq \operatorname{deg}\left(\omega_{n}^{ \pm}\right)$, which shows by the first part of Lemma 2.9 that

$$
\operatorname{dim} D_{n, \mathfrak{p}} \geq \operatorname{dim} D_{n, \mathfrak{p}}^{+}+\operatorname{dim} D_{n, \mathfrak{p}}^{-} \geq \operatorname{deg}\left(\omega_{n}^{+}\right)+\operatorname{deg}\left(\omega_{n}^{-}\right)=p^{n}
$$

Hence we have proved

$$
\omega_{n}^{\mp} D_{n, \mathfrak{p}}=D_{n, \mathfrak{p}}^{ \pm} \cong K_{\mathfrak{p}} \otimes \Lambda / \omega_{n}^{ \pm} .
$$

Corollary 2.11. We have:

(i) $H_{+}^{1}\left(D_{n, \mathfrak{p}}, V\right) \cap H_{-}^{1}\left(D_{n, \mathfrak{p}}, V\right)=0$,

(ii) $H_{+}^{1}\left(D_{n, \mathfrak{p}}, V\right)+H_{-}^{1}\left(D_{n, \mathfrak{p}}, V\right)=H_{f}^{1}\left(D_{n, \mathfrak{p}}, V\right)$.

We define the plus-minus subgroup $H_{ \pm}^{1}\left(D_{n, \mathfrak{p}}, T\right)$ (respectively $H_{ \pm}^{1}\left(D_{n, \mathfrak{p}}, W\right)$ ) as the inverse (respectively the direct) image of $H_{ \pm}^{1}\left(D_{n, \mathfrak{p}}, V\right)$. We also define $H_{ \pm}^{1}\left(D_{n, \mathfrak{p}}, T^{*}\right)$ to be the orthogonal complement of $H_{ \pm}^{1}\left(D_{n, \mathfrak{p}}, W\right)$ with respect to the local Tate pairing.

\subsection{Local ranks of universal norms}

We define $\mathcal{H}^{1}=\lim _{\leftarrow} H^{1}\left(D_{n, \mathfrak{p}}, T^{*}\right)$ and let $\mathcal{H}_{ \pm}^{1}=\lim _{ \pm} H_{ \pm}^{1}\left(D_{n, \mathfrak{p}}, T^{*}\right)$. In this section, we first prove the following proposition which is analogous to Proposition 8.1 in [30]:

Proposition 2.12. $\mathcal{H}^{1}$ is a free $\Lambda$-module of rank 2. Assuming Conjecture 2.5, both $\mathcal{H}_{ \pm}^{1}$ are free of rank 1 , and $\mathcal{H}_{+}^{1} \cap \mathcal{H}_{-}^{1}=\{0\}$.

Proof. We first verify that the $\Lambda$-module $\mathcal{H}^{1}$ is free, using an argument identical to that of Remark 2.8 in [8]. It follows from Proposition 4.2.9 in [23] that the cohomology $H^{\bullet}\left(K_{\mathfrak{p}}, T^{*} \otimes \Lambda\right)$ is represented by a perfect complex of $\Lambda$-modules (i.e., projective, hence free, $\Lambda$-modules of finite type) concentrated in degrees 0,1 and 2 . In particular, since we have $H^{2}\left(K_{\mathfrak{p}}, T^{*} \otimes \Lambda\right)=0$ by Lemma 2.1, this complex may be taken in degrees 0 and 1 . Similarly, the cohomology $H^{\bullet}\left(K_{\mathfrak{p}}, T \otimes \Lambda\right)$ is represented by a perfect complex of $\Lambda$-modules concentrated in degrees 0 and 1 . As the coefficient ring $\Lambda$ is Gorenstein, the two complexes $H^{\bullet}\left(K_{\mathfrak{p}}, T^{*} \otimes \Lambda\right)$ and $H^{\bullet}\left(K_{\mathfrak{p}}, T \otimes \Lambda\right)$ are related by the duality functor $\mathrm{RHom}_{\Lambda}(-, \Lambda)[-2]$ (see Proposition 5.2.4 in [23]). As a result, each of these two complexes is also represented by a perfect complex concentrated in degrees $2-1=1$ and $2-0=2$, hence by a single projective (hence free) $\Lambda$-module of finite type in degree 1 . This completes the proof that the $\Lambda$-module $\mathcal{H}^{1} \cong H^{1}\left(K_{\mathfrak{p}}, T^{*} \otimes \Lambda\right)$ is free.

It further follows from Lemma 2.1 that the natural projection $\mathcal{H}^{1} \rightarrow H^{1}\left(K_{\mathfrak{p}}, T^{*}\right)$ is surjective. One can verify (as in Theorem A.8 of [7]) that the $\mathcal{O}_{\mathfrak{p}}$-module $H^{1}\left(K_{\mathfrak{p}}, T^{*}\right)$ is free of rank 2 and thus the first assertion of the proposition follows.

Let $\varepsilon=+$ or - . As $H_{\varepsilon}^{1}\left(D_{n, \mathfrak{p}}, T^{*}\right)$ is the orthogonal complement of $H_{\varepsilon}^{1}\left(D_{n, \mathfrak{p}}, W\right)$ which is contained in the module $H_{f}^{1}\left(D_{n, \mathfrak{p}}, W\right)$, we see at once that $H_{f}^{1}\left(D_{n, \mathfrak{p}}, T^{*}\right)$ 
annihilates $H_{\varepsilon}^{1}\left(D_{n, \mathfrak{p}}, W\right)$ and therefore it is contained in $H_{\varepsilon}^{1}\left(D_{n, \mathfrak{p}}, T^{*}\right)$. Consider the exact sequence

$$
0 \longrightarrow H_{f}^{1}\left(D_{n, \mathfrak{p}}, T^{*}\right) \longrightarrow H_{\varepsilon}^{1}\left(D_{n, \mathfrak{p}}, T^{*}\right) \longrightarrow Q_{n} \longrightarrow 0
$$

where $Q_{n}=H_{\varepsilon}^{1}\left(D_{n, \mathfrak{p}}, T^{*}\right) / H_{f}^{1}\left(D_{n, \mathfrak{p}}, T^{*}\right)$. Passing to the limit in the sequence above (using Proposition B.1.1 in [32]), we conclude that

$\mathcal{H}_{\varepsilon}^{1} \stackrel{\sim}{\longrightarrow}{\underset{n}{n}}_{\lim } Q_{n} \cong \underbrace{\lim }_{n} \operatorname{Hom}\left(\frac{H_{f}^{1}\left(D_{n, \mathfrak{p}}, W\right)}{H_{\varepsilon}^{1}\left(D_{n, \mathfrak{p}}, W\right)}, \mathbb{Q}_{p} / \mathbb{Z}_{p}\right) \cong \operatorname{Hom}\left(\underline{\lim } \frac{H_{f}^{1}\left(D_{n, \mathfrak{p}}, W\right)}{H_{\varepsilon}^{1}\left(D_{n, \mathfrak{p}}, W\right)}, \mathbb{Q}_{p} / \mathbb{Z}_{p}\right)$

since we assumed Conjecture 2.5 and where the second isomorphism follows from local duality. Furthermore,

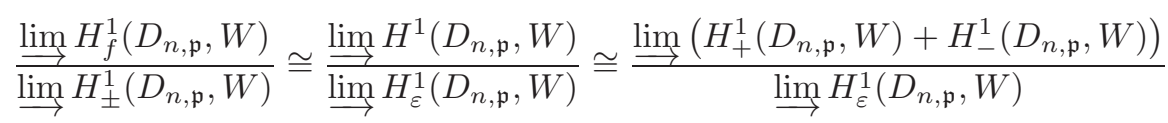

$$
\begin{aligned}
& \cong \lim _{\longrightarrow} H_{-\varepsilon}^{1}\left(D_{n, \mathfrak{p}}, W\right)
\end{aligned}
$$

where the first isomorphism follows from Conjecture 2.5 and the second and third from Lemma 2.9. That $\mathcal{H}_{\varepsilon}^{1}$ is free of rank one now follows from Remark 2.10.

To finish the proof of the proposition, observe that, by local duality,

$$
\begin{aligned}
H_{+}^{1}\left(D_{n, \mathfrak{p}}, T^{*}\right) \cap H_{-}^{1}\left(D_{n, \mathfrak{p}}, T^{*}\right) & \cong \operatorname{Hom}\left(\frac{H^{1}\left(D_{n, \mathfrak{p}}, W\right)}{H_{+}^{1}\left(D_{n, \mathfrak{p}}, W\right)+H_{-}^{1}\left(D_{n, \mathfrak{p}}, W\right)}, \mathbb{Q}_{p} / \mathbb{Z}_{p}\right) \\
& \cong \operatorname{Hom}\left(\frac{H^{1}\left(D_{n, \mathfrak{p}}, W\right)}{H_{f}^{1}\left(D_{n, \mathfrak{p}}, W\right)}, \mathbb{Q}_{p} / \mathbb{Z}_{p}\right) .
\end{aligned}
$$

Passing to the limit, the final assertion of the proposition now follows from Conjecture 2.5.

As in [30] (see Conjecture 2.2), we conjecture that the following holds.

Conjecture 2.13. $\mathcal{H}^{1} \cong \mathcal{H}_{+}^{1} \oplus \mathcal{H}_{-}^{1}$.

It would be interesting to know if Conjecture 2.13 follows from the known cases (see Theorem 8.4 in [30]) in weight 2 via a variant of Conjecture 2.7.

\subsection{Definitions of the Selmer groups}

For every finite extension $F$ of $K$ and for every $v \nmid p$ of $F$, let $F_{v}^{u r}$ be the maximal unramified extension of $F_{v}$. Define

$$
H_{f}^{1}\left(F_{v}, T^{*}\right)=\operatorname{ker}\left(H^{1}\left(F_{v}, T^{*}\right) \longrightarrow H^{1}\left(F_{v}^{\mathrm{ur}}, V^{*}\right)\right),
$$

and let $H_{f}^{1}\left(F_{v}, W\right)$ be the orthogonal complement of $H_{f}^{1}\left(F_{v}, T^{*}\right)$ with respect to the local Tate pairing. We then define the following Selmer groups for $X=W, T^{*}$ :

- The relaxed Selmer group

$$
\operatorname{Sel}_{\mathrm{rel}}(F, X)=\operatorname{ker}\left(H^{1}(F, X) \longrightarrow \prod_{v \nmid p} \frac{H^{1}\left(F_{v}, X\right)}{H_{f}^{1}\left(F_{v}, X\right)}\right)
$$


- The true Selmer group

$$
\operatorname{Sel}(F, X)=\operatorname{ker}\left(H^{1}(F, X) \longrightarrow \prod \frac{H^{1}\left(F_{v}, X\right)}{H_{f}^{1}\left(F_{v}, X\right)}\right) .
$$

- The strict Selmer group

$$
\operatorname{Sel}_{\text {str }}(F, X)=\operatorname{ker}\left(H^{1}(F, X) \longrightarrow \prod_{v \nmid p} \frac{H^{1}\left(F_{v}, X\right)}{H_{f}^{1}\left(F_{v}, X\right)} \times \prod_{v \mid p} H^{1}\left(F_{v}, W\right)\right) .
$$

- For fields $F$ where the plus-minus subgroups are defined, the \pm -Selmer group

$$
\operatorname{Sel}_{ \pm}(F, X)=\operatorname{ker}\left(H^{1}(F, X) \longrightarrow \prod_{v \nmid p} \frac{H^{1}\left(F_{v}, X\right)}{H_{f}^{1}\left(F_{v}, X\right)} \times \prod_{v \mid p} H_{ \pm}^{1}\left(F_{v}, X\right)\right) .
$$

Note the obvious inclusions

$$
\operatorname{Sel}_{\mathrm{str}}(F, W) \subset \operatorname{Sel}_{ \pm}(F, W) \subset \operatorname{Sel}(F, W) \subset \operatorname{Sel}_{\mathrm{rel}}(F, W),
$$

and similarly with $W$ replaced by $T^{*}$. If $F / K$ is an infinite extension, we define

$$
\operatorname{Sel}_{*}(F, W)=\underset{\lim }{\longrightarrow} \operatorname{Sel}_{*}\left(F^{\prime}, W\right), \quad \check{S}_{*}\left(F, T^{*}\right)=\lim _{\longleftarrow} \operatorname{Sel}_{*}\left(F^{\prime}, T^{*}\right),
$$

where limits are taken with respect to the restriction and corestriction maps (over all finite subfields $F^{\prime}$ of $F / K$ ) respectively.

We remark that the Selmer group denoted by $\operatorname{Sel}\left(f / D_{n}\right)$ in the statement of Theorem A (respectively $\operatorname{Sel}_{ \pm}\left(f / D_{\infty}\right)$ in Theorem B) in the introduction is $\operatorname{Sel}\left(D_{n}, W\right)$ (respectively $\operatorname{Sel}_{ \pm}\left(D_{\infty}, W\right)$ ) above.

\section{Elliptic units and a nontrivial anticyclotomic Euler system}

Let $\mathfrak{a}$ be an integral ideal of $\mathcal{O}_{K}$ coprime to $6 p \mathfrak{f}$, and write $\mathcal{K}_{\mathfrak{a}}$ for the union of all ray class fields of $K$ of conductor prime to $\mathfrak{a}$. Let $c_{\text {ell, }}$ denote the Euler system of elliptic units for $\left(Z_{p}(1), \mathfrak{f} p, \mathcal{K}_{\mathfrak{a}}\right)$ as in [32]. Let

$$
\psi_{\mathfrak{p}}: G_{K} \longrightarrow \operatorname{Aut}_{\mathcal{O}_{K}}\left(V_{f} / T_{f}\right) \cong \mathcal{O}_{\mathfrak{p}}^{\times}
$$

be the $p$-adic avatar of the Hecke character associated to $f$ by the theory of $\mathrm{CM}$ and let

$$
\psi_{\mathfrak{p}}^{(w / 2)}=\psi_{\mathfrak{p}} \otimes \chi_{\text {cyc }}^{\omega / 2}: G_{K} \longrightarrow \operatorname{Aut}_{\mathcal{O}_{K}}(W)
$$

be its central critical twist as in Theorem 14.2 of [17]. Following [32], §6, one may twist the Euler system $c_{\text {ell } \mathfrak{a}}$ by the character $\psi_{\mathfrak{p}}^{-1} \otimes \chi_{\text {cyc }}^{-\omega / 2}$ to obtain an Euler system for $\left(T^{*}, \mathfrak{f} p, \mathcal{K}_{\mathfrak{a}}\right)$. Then $c_{\mathfrak{a}}(F) \in \operatorname{Sel}_{\text {rel }}\left(F, T^{*}\right)$ for every finite extension $F \subset \mathcal{K}_{\mathfrak{a}}$ of $K$. Let $K_{\infty}$ denote the unique $\mathbb{Z}_{p}^{2}$-extension of $K$. Set

$$
c_{\mathfrak{a}}(L)=\left\{c_{\mathfrak{a}}\left(F^{\prime}\right)\right\} \in \check{S}_{\mathrm{rel}}\left(L, T^{*}\right)
$$

for any extension $L \subset K_{\infty}$, where the inverse limit is over all subfields $F^{\prime}$ of $L$ that are finite over $K$. Let $\mathcal{C}_{\mathfrak{a}}(F)$ denote the $\mathcal{O}_{\mathfrak{p}}[[\operatorname{Gal}(F / K)]]$-submodule of $\operatorname{Sel}_{\text {rel }}\left(F, T^{*}\right)$ generated by $c_{\mathfrak{a}}(F)$ and $\mathcal{C}(F)$ the submodule generated by $\mathcal{C}_{\mathfrak{a}}(F)$ as $\mathfrak{a}$ varies over 
all ideals of $\mathcal{O}_{K}$ coprime to $6 p \mathfrak{f}$. Recall that $\psi=\bar{\psi} \circ \tau$ (where $\tau \in \operatorname{Gal}(K / \mathbb{Q})$ is the nontrivial automorphism) so that the sign of the functional equation for the Hecke $L$-function of $\psi$ makes sense and equals $W(\psi)= \pm 1$. Note that this is the sign of the functional equation for $L(f, s)$ as well.

The following is the analogue of Proposition 3.1 in [1] in our setting. Note however that since the higher-weight versions of the nonvanishing results of Rohrlich [28] are not available, we adapt Arnold's approach (see [2]) in order to verify the nonvanishing of $\mathcal{C}\left(D_{\infty}\right)$ :

Proposition 3.1. The image of $\mathcal{C}\left(D_{\infty}\right)$ in $\mathcal{H}^{1}$ is nontrivial. Furthermore, assuming the truth of Conjecture 2.13, the image of $\mathcal{C}\left(D_{\infty}\right)$ in $\mathcal{H}^{1}$ is contained in $\mathcal{H}_{\varepsilon}^{1}$ if and only if $\varepsilon$ is the sign of $W(\psi)$.

Proof. For a character $\phi$ of $\Gamma$, let the composition

$$
\mathrm{Tw}_{\phi}: \lim _{\longleftarrow} H^{1}\left(D_{n}, T^{*}\right) \stackrel{\sim}{\longrightarrow} \lim _{\longleftarrow} H^{1}\left(D_{n}, T^{*} \otimes \phi\right) \longrightarrow H^{1}\left(K, T^{*} \otimes \phi\right)
$$

denote the twisting map. We will choose $\phi$ suitably so that $\operatorname{Tw}_{\phi}\left(c_{\mathfrak{a}}\left(D_{\infty}\right)\right)$ has nontrivial image in $H^{1}\left(K_{\mathfrak{p}}, T^{*} \otimes \phi\right)$.

By mimicking the the proof of Proposition 2.3 in [2] (which in turn relies on [13]), one may find an integer $d$ so that:

- The character $\left(\psi_{\mathfrak{p}} / \bar{\psi}_{\mathfrak{p}}\right)^{d}$ factors through $\Gamma$,

- if $W(\psi)=1$, then for $\psi^{*}=\psi^{2 d+1}$ and $\omega^{*}=(2 d+1)(\omega-1)+1=2 d(\omega-1)+\omega$, we have $L\left(\psi^{*}, \omega^{*} / 2\right) \neq 0$.

- if $W(\psi)=-1$, then for $\psi_{*}=\psi^{2 d-1}$ and $\omega_{*}=(2 d-1)(\omega-1)+1=$ $2 d(\omega-1)+2-\omega$, we have $L\left(\psi_{*}, \omega_{*} / 2\right) \neq 0$

Assume first that $W(\psi)=1$ and let $\Psi=\psi^{*}, \boldsymbol{\omega}=\omega^{*}$ and $\left.\phi=\left(\psi_{\mathfrak{p}} / \bar{\psi}_{\mathfrak{p}}\right)^{d}\right)$. Note that $\phi$ factors through $\Gamma$ by our choices and running assumptions. Define $T_{\Psi}$ (respectively $T_{\Psi}^{*}$ ) to be the free $\mathcal{O}_{\mathfrak{p}}$-module of rank one on which $G_{K}$ acts by $\Psi_{\mathfrak{p}} \otimes \chi_{\text {cyc }}\left(\right.$ respectively $\left.\Psi_{\mathfrak{p}}^{-1}\right)$. As in [20], let $z^{\text {Kato }} \in \lim H^{1}\left(K\left(\boldsymbol{\mu}_{p^{n}}\right)_{\mathfrak{p}}, T_{\Psi}^{*}\right)$ be the element obtained from Kato's Euler system. Then,

$$
\mathrm{Col}^{ \pm}\left(z^{\text {Kato }}\right)=L_{p}^{ \pm}
$$

where $\mathrm{Col}^{ \pm}$are the plus-minus Coleman maps defined in Section 3.4 of [20] and $L_{p}^{ \pm}$ are the plus-minus cyclotomic $p$-adic $L$-functions attached to the theta series of the Hecke character $\Psi$, defined as in the displayed equations (4) and (5) of [20]. The interpolation property of the $p$-adic $L$-function (see [20] and [24]) then shows that

$$
\chi_{\text {cyc }}^{\boldsymbol{\omega} / 2-1}\left(\mathrm{Col}^{+}\left(z^{\text {Kato }}\right)\right)=\chi_{\text {cyc }}^{\boldsymbol{\omega} / 2-1}\left(L_{p}^{+}\right)=\Omega_{\Psi}^{-1} L(\Psi, \omega / 2) \neq 0 .
$$

where $\Omega_{\Psi}$ is a nonzero complex number whose exact value we need not know. Let $V_{\Psi}=T_{\Psi} \otimes \mathbb{Q}_{p}$ ( similarly $V_{\Psi}^{*}$ ) be the associated two dimensional $\mathbb{Q}_{p}$-vector space. For $\eta_{+} \in D\left(V_{\Psi}^{*}\right)$ defined as in [20], §3.5.1, one constructs the extended 
$\operatorname{logarithm} \mathcal{L}_{\eta^{+}}$(see Section 3.2 of [20] for a precise definition) making use of PerrinRiou's map [26] as well as a logarithm map $\log _{p, \omega}^{+}$defined in [24]. Then

$$
\mathrm{Col}^{+}\left(z^{\text {Kato }}\right)=\mathcal{L}_{\eta^{+}}\left(z^{\text {Kato }}\right) / \log _{p, \boldsymbol{w}}^{+},
$$

which in turn shows, thanks to $(3.1)$, that $\underset{\chi_{\text {cyc }}^{\omega / 2-1}}{\sim}\left(\mathcal{L}_{\eta^{+}}\left(z^{\text {Kato }}\right)\right) \neq 0$.

To simplify notation (and facilitate the comparison of our arguments to those of Lei), we set $r=\omega / 2-1$. Let $z_{-r}$ be the image of $z^{\text {Kato }}$ under the composition

$$
\lim _{n} H^{1}\left(K\left(\boldsymbol{\mu}_{p^{n}}\right)_{\mathfrak{p}}, T_{\Psi}^{*}\right) \stackrel{(-1)^{r} \mathrm{Tw}_{\chi_{\text {cyc }}^{-r}}^{\longrightarrow}}{\longrightarrow} H^{1}\left(K_{\mathfrak{p}}, T_{\Psi}^{*}(-r)\right) \text {. }
$$

Let $M^{\mathfrak{D}}=\operatorname{Hom}\left(M, \mathbb{Q}_{p}\right)$ (respectively $\left.M^{*}=M^{\mathfrak{D}}(1)\right)$ stand for the linear dual (respectively Cartier dual) of a $\mathbb{Q}_{p}$-vector space $M$. Note then that $V_{\Psi}^{*}(-r) \cong$ $V_{\Psi}(r+1)^{\mathfrak{D}}(1) \cong V_{\Psi}(r+1)^{*}$. Let

$$
\exp _{r+1}^{*}: H^{1}\left(K_{\mathfrak{p}}, V_{\Psi}(r+1)^{*}\right) \longrightarrow D^{0}\left(V_{\Psi}(r+1)^{*}\right)
$$

be the dual exponential map and let [, ] be the natural pairing on $D\left(V_{\Psi}(r+1)\right) \otimes$ $D\left(V_{\Psi}(r+1)^{*}\right)$. Kurihara's calculation [19] yields

$$
\chi_{\text {cyc }}^{r}\left(\mathcal{L}_{\eta^{+}}\left(z^{\text {Kato }}\right)\right)=r !\left[\left(1-\frac{\varphi^{-1}}{p}\right)(1-\varphi)^{-1}\left(\eta_{r+1}^{+}\right), \exp _{r+1}^{*}\left(z_{-r}\right)\right],
$$

where $\eta_{r+1}^{+}$is the image of $\eta^{+}$under the canonical map $D\left(V_{\Psi}\right) \rightarrow D\left(V_{\Psi}(r+1)\right)$. This shows that $z_{-r} \neq 0$, as we have verified above that the expression on the left of (3.2) is nonzero.

Similarly to above, we define $c_{\mathfrak{a}}^{\Psi}\left(K\left(\boldsymbol{\mu}_{p^{\infty}}\right)\right) \in \check{S}_{\text {rel }}\left(K\left(\boldsymbol{\mu}_{p^{\infty}}\right), T_{\Psi}^{*}\right)$ and observe that the image of $c_{\mathfrak{a}}^{\Psi}\left(K\left(\boldsymbol{\mu}_{p} \infty\right)\right)$ under the composition

$$
\lim _{\longleftarrow} H^{1}\left(K\left(\boldsymbol{\mu}_{p^{n}}\right), T_{\Psi}^{*}\right) \longrightarrow \lim _{\longleftarrow} H^{1}\left(K\left(\boldsymbol{\mu}_{p^{n}}\right)_{\mathfrak{p}}, T_{\Psi}^{*}\right) \stackrel{(-1)^{r} \mathrm{Tw}_{\chi_{\text {cyc }}^{-r}}^{\longrightarrow}}{\longrightarrow} H^{1}\left(K_{\mathfrak{p}}, T_{\Psi}(\boldsymbol{\omega} / 2)^{*}\right)
$$

agrees with the image of $c_{\mathfrak{a}}\left(D_{\infty}\right)$ under the composition

$$
\lim _{\longleftarrow} H^{1}\left(D_{n}, T^{*}\right) \longrightarrow \lim _{\longleftarrow} H^{1}\left(D_{n, \mathfrak{p}}, T^{*}\right) \stackrel{(-1)^{r} \mathrm{Tw}_{\phi}}{\longrightarrow} H^{1}\left(K_{\mathfrak{p}}, T_{\Psi}(\boldsymbol{\omega} / 2)^{*}\right) .
$$

Furthermore, 15.6.1 in [17] shows that the image of $c_{\mathfrak{a}}^{\Psi}\left(K\left(\boldsymbol{\mu}_{p}\right)\right)$ under (3.3) agrees with $z_{-r}$ (up to a nonzero factor), in particular the image of $c_{\mathfrak{a}}\left(D_{\infty}\right)$ inside $\mathcal{H}^{1}$ is nonzero. This completes the proof of the first part of the proposition when $W(\psi)=1$. In case $W(\psi)=-1$, we replace $\phi$ by $\phi \circ \tau$ and reduce similarly to the nonvanishing of $L\left(\psi_{*}, w_{*} / 2\right)$.

The second part may be now proved mimicking the proof of Proposition 3.1 in [1]. Let $\varepsilon$ denote the sign of $W(\psi)$. For any positive integer $n$,

(a) Greenberg's formula (see [12], page 247) for the sign of the functional equation $W(\chi \psi)$ shows that $L(\chi \psi, \omega / 2)=0$ for all characters $\chi \in \Xi_{n}^{\varepsilon}$. We remark that Greenberg verifies his formula only for weight 2 modular forms in [12], 
but his proof easily generalizes to the case of interest here using Weil's formula for root numbers, stated as in Proposition 2.4 of [2].

(b) Kato's generalized explicit reciprocity law (see [16], [17] and Proposition 7.8 in [20], where the latter may be generalized using Lemma 2.4 to cover characters of $\Gamma$ ) relates the localization of the elliptic units to the special value $L(\chi \psi, \omega / 2)$. For every character $\chi \in \Xi_{n}^{\varepsilon}$, the reciprocity law and the vanishing of $L(\chi \psi, \omega / 2)$ shows that the image $\mathcal{C}\left(D_{n, \mathfrak{p}}\right) \subset H^{1}\left(D_{n, \mathfrak{p}}, T^{*}\right)$ of $\mathcal{C}\left(D_{\infty}\right)$ annihilates the $\chi$-part of $H_{f}^{1}\left(D_{n, \mathfrak{p}}, W\right)$. We therefore infer that $\mathcal{C}\left(D_{n, \mathfrak{p}}\right)$ annihilates $H_{\varepsilon}^{1}\left(D_{n, \mathfrak{p}}, W\right)$. Hence $\mathcal{C}\left(D_{n, \mathfrak{p}}\right) \subset H_{\varepsilon}^{1}\left(D_{n, \mathfrak{p}}, T^{*}\right)$. (Note that $H_{\varepsilon}^{1}\left(D_{n, \mathfrak{p}}, T^{*}\right)$ is the orthogonal complement of $H_{\varepsilon}^{1}\left(D_{n, \mathfrak{p}}, W\right)$ by its very definition.) This shows that the image of $\mathcal{C}\left(D_{\infty}\right)$ in $\mathcal{H}^{1}$ belongs to $\mathcal{H}_{\varepsilon}^{1}$ and the the proof of the proposition follows since we assumed Conjecture 2.13.

Remark 3.2. The proof of Proposition 3.1 is the only place where we need Conjecture 2.13 in an essential way in order to prove our main result (that is, Theorem 4.1). If the nonvanishing results of Rohrlich were available in our setting, we could then follow the proof of Proposition 3.1 in [1] and show directly that the image of $\mathcal{C}\left(D_{\infty}\right)$ in $\mathcal{H}^{1}$ does not belong to $\mathcal{H}_{-\varepsilon}^{1}$, without any need of Conjecture 2.13.

Definition 3.3. We define $X_{*}\left(D_{\infty}, W\right)=\operatorname{Hom}_{\mathbb{Z}_{p}}\left(\operatorname{Sel}_{*}\left(D_{\infty}\right), \mathbb{Q}_{p} / \mathbb{Z}_{p}\right)$ for $*=$ $\{$ rel, str,,$\pm \varnothing\}$. We adopt the convention of [1] that $\Lambda$ acts on $X_{*}\left(D_{\infty}, W\right)$ by the rule $\lambda \cdot f(x)=f\left(\lambda^{\iota} x\right)$, where $\iota: \Lambda \rightarrow \Lambda$ is the involution on $\Lambda$ induced by inversion on group-like elements.

\section{Proposition 3.4.}

(i) The $\Lambda$-module $\check{S}_{\text {rel }}\left(D_{\infty}, T^{*}\right)$ (respectively $X_{\text {str }}\left(D_{\infty}, W\right)$ ) is torsion-free of rank one (respectively torsion).

(ii) The $\Lambda$-module $X_{\text {rel }}\left(D_{\infty}, W\right)$ has rank one.

(iii) We have

$$
\operatorname{char}\left(X_{\mathrm{str}}\left(D_{\infty}, W\right)\right)=\operatorname{char}\left(\check{S}_{\mathrm{rel}}\left(D_{\infty}, T^{*}\right) / \mathcal{C}\left(D_{\infty}\right)\right)
$$

Proof. (i) can be proved using the Euler system machinery as in [32], Section II.3, and the nontriviality of $\mathcal{C}\left(D_{\infty}\right)$. (ii) follows from Poitou-Tate global duality as in Proposition 4.2.3 of [25], and Lemma 2.1. Part (iii) follows from the 2-variable main conjecture for $K$ (see Theorem 4.1 (i) in [31]) exactly as in Section 3.3 of [2].

\section{Theorem 3.5.}

(i) $\operatorname{rank}_{\Lambda}\left(\check{S}_{ \pm}\left(D_{\infty}, T^{*}\right)\right)=\operatorname{rank}_{\Lambda}\left(X_{ \pm}\left(D_{\infty}, W\right)\right)$.

(ii) Assuming that Conjecture 2.13 holds true, $X_{\varepsilon}\left(D_{\infty}, W\right)$ has $\Lambda$-rank one and $X_{-\varepsilon}\left(D_{\infty}, W\right)$ is $\Lambda$-torsion, where $\varepsilon$ is the sign of $W(\psi)$. 
Remark 3.6. Note in particular that, it follows from Theorem 3.5 and the fact that $\check{S}_{\text {rel }}\left(D_{\infty}, T^{*}\right)$ is torsion-free (Proposition 3.4) that $\check{S}_{\text {str }}\left(D_{\infty}, T^{*}\right)=\check{S}_{-\varepsilon}\left(D_{\infty}, T^{*}\right)=0$, assuming Conjecture 2.13.

Proof of Theorem 3.5. Poitou-Tate global duality yields an exact sequence

$$
\begin{aligned}
0 \rightarrow \check{S}_{ \pm}\left(D_{\infty}, T^{*}\right) \rightarrow \check{S}_{\mathrm{rel}}\left(D_{\infty}, T^{*}\right) & \rightarrow \mathcal{H}^{1} / \mathcal{H}_{ \pm}^{1} \\
& \rightarrow X_{ \pm}\left(D_{\infty}, W\right) \rightarrow X_{\mathrm{str}}\left(D_{\infty}, W\right) \rightarrow 0
\end{aligned}
$$

which proves (i) using Propositions 2.12 and 3.4. Assuming the truth of Conjecture 2.13, we note that $\mathcal{C}\left(D_{\infty}\right) \subset \check{S}_{\varepsilon}\left(D_{\infty}, T^{*}\right)$ by Proposition 3.1. The rest can be proved following the proof of Theorem 3.6 in [1] verbatim.

\section{Applications}

\subsection{The variation of Selmer ranks}

In [1], §5, the authors obtain the anticyclotomic analogues of Kobayashi's control theorems (Theorem 9.3 in [18]). These extend without further difficulty to apply in our setting and may be used along with Theorem 3.5 to prove the following result on the variation of the ranks of Selmer ranks along the anticyclotomic tower.

Let $Y_{ \pm}$denote the $\Lambda$-torsion submodule of $X_{ \pm}\left(D_{\infty}, W\right)$.

Theorem 4.1. Let $\varepsilon$ denote the sign of $W(\psi)$. We assume the truth of Conjectures 2.5 and 2.13. Then, for all $n$,

$$
\operatorname{corank}_{\mathcal{O}_{\mathfrak{p}}}\left(\operatorname{Sel}\left(D_{n}, W\right)\right)=\operatorname{rank}_{\mathcal{O}_{\mathfrak{p}}}\left(\Lambda / \omega_{n}^{\varepsilon}\right)+\operatorname{rank}_{\mathcal{O}_{\mathfrak{p}}}\left(Y_{+} / \omega_{n}^{+}\right)+\operatorname{rank}_{\mathcal{O}_{\mathfrak{p}}}\left(Y_{-} / \omega_{n}^{-}\right)
$$

\subsection{An anticyclotomic main conjecture}

The theorem we state next may be thought of as a form of an anticyclotomic main conjecture (without the $p$-adic $L$-function). The assumptions under which Theorem 4.1 holds are in effect here as well.

Theorem 4.2. Let $\varepsilon$ be the sign of $W(\psi)$. We then have an equality of characteristic ideals

$$
\operatorname{char}\left(X_{-\varepsilon}\left(D_{\infty}, W\right)\right)=\operatorname{char}\left(\mathcal{H}_{\varepsilon}^{1} / \mathcal{C}\left(D_{\infty}\right)\right)
$$

Proof. The exact sequence (3.5) yields an injection

$$
\check{S}_{\mathrm{rel}}\left(D_{\infty}, T^{*}\right) / \check{S}_{\varepsilon}\left(D_{\infty}, T^{*}\right) \hookrightarrow \mathcal{H}^{1} / \mathcal{H}_{\varepsilon}^{1} \cong \mathcal{H}_{-\varepsilon}^{1} .
$$

Since both $\check{S}_{\text {rel }}\left(D_{\infty}, T^{*}\right)$ and $\check{S}_{\varepsilon}\left(D_{\infty}, T^{*}\right)$ are $\Lambda$-modules of rank one (Theorem 3.5) and $\mathcal{H}_{-\varepsilon}^{1}$ is $\Lambda$-torsion free, it follows that $\check{S}_{\text {rel }}\left(D_{\infty}, T^{*}\right)=\check{S}_{\varepsilon}\left(D_{\infty}, T^{*}\right)$. Furthermore, as $\check{S}_{-\varepsilon}\left(D_{\infty}, T^{*}\right)=0$ (Remark 3.6), the exact sequence (3.5) reduces to the exact sequence

$$
0 \longrightarrow \mathcal{H}_{\varepsilon}^{1} / \check{S}_{\varepsilon}\left(D_{\infty}, T^{*}\right) \longrightarrow X_{-\varepsilon}\left(D_{\infty}, W\right) \longrightarrow X_{\text {str }}\left(D_{\infty}, W\right) \longrightarrow 0 .
$$


This combined with the exact sequence

$$
0 \longrightarrow \check{S}_{\varepsilon}\left(D_{\infty}, T^{*}\right) / \mathcal{C}\left(D_{\infty}\right) \longrightarrow \mathcal{H}_{\varepsilon}^{1} / \mathcal{C}\left(D_{\infty}\right) \longrightarrow \mathcal{H}_{\varepsilon}^{1} / \check{S}_{\varepsilon}\left(D_{\infty}, T^{*}\right) \longrightarrow 0
$$

along with Proposition 3.4 (iii) proves the theorem.

One expects that the right side of the equality in Theorem 4.2 would relate to an appropriately defined anticyclotomic $p$-adic $L$-function, via Kato's explicit reciprocity law; see Theorem 4.3 in [1] for the relevant discussion in the case $w=2$.

\section{References}

[1] Agboola, A. And Howard, B.: Anticyclotomic Iwasawa theory of CM elliptic curves. II. Math. Res. Lett. 12 (2005), no. 5-6, 611-621.

[2] Arnold, T.: Anticyclotomic main conjectures for CM modular forms. J. Reine Angew. Math. 606 (2007), 41-78.

[3] Bertolini, M. and Darmon, H.: Iwasawa's main conjecture for elliptic curves over anticyclotomic $\mathbb{Z}_{p}$-extensions. Ann. of Math. (2) 162 (2005), no. 1, 1-64.

[4] Bertolini, M.: Selmer groups and Heegner points in anticyclotomic $\mathbb{Z}_{p}$-extensions. Compositio Math. 99 (1995), no. 2, 153-182.

[5] Berger, L.: Représentations de de Rham et normes universelles. Bull. Soc. Math. France 133 (2005), no. 4, 601-618.

[6] Bloch, S. And Kato, K.: $L$-functions and Tamagawa numbers of motives. In The Grothendieck Festschrift, Vol. I, 333-400. Progr. Math. 86, Birkhäuser Boston, Boston, MA, 1990.

[7] BÜYÜKBoduk, K.: Stark units and the main conjectures for totally real fields. Compos. Math. 145 (2009), 1163-1195.

[8] BÜYÜKBODUK, K.: On Euler systems of rank $r$ and their Kolyvagin systems. Indiana Univ. Math. J. 59 (2010), no. 4, 1277-1332.

[9] Chida, M. And Hsien, M.-L.: On the anticyclotomic Iwasawa main conjecture for modular forms. To appear in Compos. Math.

[10] Deligne, P.: Formes modulaires et répresentations $\ell$-adiques. In Séminaire Bourbaki Vol. 1968/69, Exp. No. 355, 139-172. Lecture Notes in Math. 175, Springer, Berlin, 1971.

[11] Darmon, H. And Iovita, A.: The anticyclotomic main conjecture for elliptic curves at supersingular primes. J. Inst. Math. Jussieu 7 (2008), no. 2, 291-325.

[12] Greenberg, R.: On the Birch and Swinnerton-Dyer conjecture. Invent. Math. 72 (1983), no. 2, 241-265.

[13] Greenberg, R.: On the critical values of Hecke $L$-functions for imaginary quadratic fields. Invent. Math. 79 (1985), no. 1, 79-94.

[14] Howard, B.: The Heegner point Kolyvagin system. Compos. Math. 140 (2004), no. $6,1439-1472$.

[15] HowARD, B.: Iwasawa theory of Heegner points on abelian varieties of $\mathrm{GL}_{2}$ type. Duke Math. J. 124 (2004), no. 1, 1-45. 
[16] Kato, K.: Generalized explicit reciprocity laws. Algebraic number theory (Hapcheon/Saga, 1996) Adv. Stud. Contemp. Math. (Pusan) 1 (1999), 57-126.

[17] Kato, K.: p-adic Hodge theory and values of zeta functions of modular forms. Cohomologies p-adiques et applications arithmétiques. III. Astérisque 295 (2004), ix, 117-290.

[18] Kobayashi, S.: Iwasawa theory for elliptic curves at supersingular primes. Invent. Math. 152 (2003), no. 1, 1-36.

[19] Kurihara, M.: On the Tate Shafarevich groups over cyclotomic fields of an elliptic curve with supersingular reduction. I. Invent. Math. 149 (2002), no. 1, 195-224.

[20] LeI, A.: Iwasawa theory for modular forms at supersingular primes. Compos. Math. 147 (2011), no. 3, 803-838.

[21] Longo, M.: Anticyclotomic Iwasawa's main conjecture for Hilbert modular forms. Comment. Math. Helv. 87 (2012), no. 2, 303-353.

[22] Nekovár̆, J.: Kolyvagin's method for Chow groups of Kuga-Sato varieties. Invent. Math. 107 (1992), no. 1, 99-125.

[23] Nekovár̆, J.: Selmer complexes. Astérisque 310 (2006), viii+559.

[24] Pollack, R.: On the $p$-adic $L$-function of a modular form at a supersingular prime. Duke Math. J. 118 (2003), no. 3, 523-558.

[25] Perrin-Riou, B.: Théorie d'Iwasawa et hauteurs p-adiques. Invent. Math. 109 (1992), no. 1, 137-185.

[26] Perrin-Riou, B.: Théorie d'Iwasawa des représentations p-adiques sur un corps local. With an appendix by Jean-Marc Fontaine. Invent. Math. 115 (1994), no. 1, 81-161.

[27] Perrin-Riou, B.: Représentations $p$-adiques et normes universelles. I. Le cas cristallin. J. Amer. Math. Soc. 13 (2000), no. 3, 533-551 (electronic).

[28] Rohrlich, D. E.: On $L$-functions of elliptic curves and anticyclotomic towers. Invent. Math. 75 (1984), no. 3, 383-408.

[29] Rubin, K.: Elliptic curves and $\mathbb{Z}_{p}$-extensions. Compositio Math. 56 (1985), no. 2, $237-250$.

[30] Rubin, K.: Local units, elliptic units, Heegner points and elliptic curves. Invent. Math. 88 (1987), no. 2, 405-422.

[31] Rubin, K.: The "main conjectures" of Iwasawa theory for imaginary quadratic fields. Invent. Math. 103 (1991), no. 1, 25-68.

[32] Rubin, K.: Euler systems. Annals of Mathematics Studies 147, Hermann Weyl Lectures, The Institute for Advanced Study, Princeton Univ. Press, Princeton, NJ, 2000.

Received January 29, 2013; revised July 4, 2013.

KÂZIm BÜYÜkboduk: Koç University, Mathematics, Rumeli Feneri Yolu, 34450 Sariyer, Istanbul, Turkey; and PUC Facultad de Matemáticas, Avenida Vicuña Mackenna 4860, Santiago, Chile.

E-mail: kbuyukboduk@ku.edu.tr

This work was partially supported by the European Commission FP7, FONDECYT and TÜBİTAK. 